\title{
Formation and Migration Energies of Interstitials \\ in Silicon Under Strain Conditions
}

\author{
Timur Halicioglu \\ Thermosciences Institute \\ NASA, Ames Research Center, M.S. 230-3 \\ Moffett Field, California 94035 \\ and \\ David M. Barnett \\ Stanford University \\ Department of Materials Science and Engineering \\ Stanford, CA 94305-2205
}

\begin{abstract}
Simulation calculations are conducted for $\mathrm{Si}$ substrates to analyze formation and diffusion energies of interstitials under strain condition using statics methods based on a Stillinger-Weber type potential function. Defects in the vicinity of the surface region and in the bulk are examined, and the role played by compressive and tensile strains on the energetics of interstitials is investigated. Results indicate that strain alters defect energetics which, in turn, modifies their diffusion characteristics.
\end{abstract}




\section{Introduction}

Diffusion of point defects plays an important role in semiconductor device fabrication technology, so that an understanding of diffusion characteristics of interstitials in semiconducting materials under strain is highly desireable [1]. Presently, little is known about the effect of strain on point defect migration properties [2]. The ability to control strain states may provide additional possibilities for controlling diffusion related phenomena in semiconductors. Recently, in several experimental studies the role played by strained surface layers on nucleation processes of defects has been investigated [3]. However, there is still no consensus about the real mechanism for the formation of defects within the surface region and the effect of strain upon it. Experiments on intrinsic point defects have produced conflicting results [4].

In this work we investigated the effect of strain on the energetics and diffusion characteristics of $\mathrm{Si}$ interstitials. Furthermore, calculations are conducted to analyze variations in the interstitial formation energy and in the diffusion barrier height as a function of distance from the exposed surface.

\section{Calculations}

Energy calculations were carried out employing the empirical potential function developed by Stillinger and Weber for systems containing Si species. This function has been used successfully in various occasions producing acceptable results $[5,6]$. Calculations were performed employing a molecular statics method representing the low temperature limit, $\mathrm{T} \Rightarrow 0 \mathrm{~K}$. The computational cell used in this study consisted of 16 layers containing $16 \mathrm{Si}$ atoms per layer. The bottom two layers were held rigid and a two-dimensional periodic boundary condition, PBC, was imposed for continuity in simulations involving surface regions. An exposed $\mathrm{Si}(100)-(2 \times 1)$ surface was considered in all computations. A three-dimensional PBC was applied for 
simulating bulk configurations. In the computational cell the $\mathrm{z}$-direction is taken perpendicular to the exposed $(100)-(2 \times 1)$ surface, while the $x$ - and $y$-directions are parallel and perpendicular to the surface dimer rows, respectively. In all cases, the diffusion of the interstitial atom is taken parallel to the $x$-direction (this represents the 11 direction along the dimer rows). Strain conditions were imposed unilaterally by elongating or compressing the bulk lattice in the $x$-, $y$ - and $z$-directions. Calculations include up to $2 \%$ compression and up to $3 \%$ tension.

Interstitial formation energies are calculated as

$$
e_{f}=E_{n+1}-\frac{n+1}{n} \cdot E_{n}
$$

where, $e_{f}$ denotes the interstitial formation energy, $E_{n+1}$ represents the total optimized energy of the system containing $n+1$ particles, including the interstitial atom, and $E_{n}$ is the total optimized energy of defect free system with $n$ atoms. The barrier height, $\Delta E$, along the diffusion path is calculated as [7]

$$
\Delta E=e_{f}(S)-e_{f}(T)
$$

Here, $e_{f}(S)$ and $e_{f}(T)$ represent interstitial energies at the saddle point $(S)$ and at the tetragonal site $(T)$, respectively. The Stillinger-Weber potential used in this study produces the tetragonal site as the energetically more favorable interstitial site in the $\mathrm{Si}$ crystal [5]. The hexagonal site (H), in this case, coincides with the saddle point along the diffusion path, which takes place via tetragonal $\rightarrow$ hexagonal $\rightarrow$ tetragonal sites in the crystal. Figure 1 depicts $3 D$ representations of tetragonal and hexagonal sites.

Per cent variations in energy values, $P_{E}$, due to applied strains are calculated as:

$$
P_{E}=100 \cdot\left(e_{f} / e_{f}^{\circ}-1\right)
$$

where, $e_{f}$ and $e_{f}^{0}$ represent interstitial energies for strained and unstrained systems, respectively. In a similar way, the per cent variation in the barrier height, $P_{E}(B)$ 
is estimated as:

$$
P_{E}(B)=100 \cdot\left(\Delta E / \Delta E^{\circ}-1\right)
$$

with $\Delta E$ and $\Delta E^{\circ}$ denoting barrier heights for strained and unstrained cases.

Figure 2 shows a side view ( $x z$ plane) of the Si crystal depicting the $\mathrm{T}$ and $\mathrm{H}$ sites, and the diffusion path in the general $x$ direction. This path represents the energetically lowest path and it was determined by moving the interstitial atom in the $x$ direction with incremental steps from its equilibrium (T) site. After every displacement, the system was completely equilibrated provided that the moving interstitial atom was forced to remain within the plane perpendicular to the diffusion path. The path is a straight line for the T-H-T portion which makes an angle of about 35 degrees angle with the $x$-axis. All the $\mathrm{T}$ and $H$ sites depicted in Figure 2 are in the same $x z$ plane for the diffusing interstital along the $x$-direction.

\section{Results and Discussions}

Figure 3 shows the per cent variation in the formation energy of a tetragonalsite interstitial as a function of applied strain. These results indicate that the interstitial formation process is more favorable under tension and it becomes energetically less favorable under compression. The percentage variation in the interstital energy is almost linear for strains applied in the z-direction. Due to symmetry, both tension and compression applied in $x$ - and $y$-directions show an almost identical variation in $P_{E}$. The general trend that we obtained here is consistent with Antonelli and Bernholc's results for pressure effects on the formation energy of T-type interstitials in Si [8].

Variation in the formation energy of an H-site interstitial is shown in Figure 4 as a function of the applied strain. Similar to the tetragonal site, the interstitial formation process is more favorable under tension and it becomes energetically less favorable with increasing compression. In this case, as anticipated, variations in the 
$P_{E}$ value differ depending on the applied strain directions (i.e. $x$ - or $y$-directions).

Per cent variations in calculated energy barrier for interstitial diffusion taking place under varying external strains are shown in Figure 5. These results indicate that strain applied in the $y$-direction has almost no effect on $\Delta E$. In both $x$ - and $y$-directions the applied tension increases $P_{E}(B)$, impeding the diffusion, and the compression lowers $P_{E}(B)$, facilitating the interstitial diffusion process. These results are consistent with the pressure effect investigations of Antonelli and Bernholc [8] on diffusion energies of interstitials in silicon. This outcome is also in agreement with the experimental findings of Kringhoj et al [9] on the diffusion of Sb interstitials, who concluded that diffusivity is enhanced by compressive strain and retarded by tensile strain (also see Ref. [1]). Furthermore, experimental findings by Zhao, et al. [10] reveal that boron diffusion in silicon is enhanced with increasing pressure; these findings also are qualitatively in agreement with present results.

Relative interstitial formation energies (for T-sites) are depicted in Figure 6 as a function of the distance, $d_{z}$ from the exposed surface. Results indicate that $e_{f}$ approaches its bulk value for interstitials deeper than $d_{z} \approx 10 \AA$. For $d_{z}$ values between approximately 5 to $10 \AA$, the deviation in $e_{f}(z) / e_{f}(b u l k)$ is rather small. For $d_{z}$ values less than $5 \AA$, however, the relative interstitial formation energy diminishes considerably as the interstitial approaches to the exposed surface region. This is partially because of the surface reconstruction which alters the overall geometry within the top surface layers. Present results, in agreement with earlier work [5], indicate that in addition to the dimerization process of top layer atoms, considerable layer-by-layer displacements take place within the top several surface layers. The first interlayer spacing (which is the distance between the top and the second layer atoms) contracts more than $8 \%$ with respect to bulk interlayer spacing. The second interlayer spacing, on the other hand, expands about $1.4 \%$, and the third interlayer spacing contracts somewhat less than $1 \%$. As a result, within the first and second interlayer spacings in particular, geometric features of $\mathrm{T}$ and $\mathrm{H}$ sites 
are distorted. Therefore, the interstitial formation energies for these sites deviate considerably from their bulk values, which in turn affect diffusion characteristics of interstitials.

Variations in the barrier height (with respect to the bulk value) are shown in Figure 7 as a function of $\mathrm{d}_{z}$. Calculated $\Delta E$ values for diffusion display a steady increase as the defect approaching the exposed surface region. This outcome indicates that interstitial diffusion within the top few layers is not favored energetically. Values for the top three interlayer spacings are not shown in the graph. It appears that the diffusion process via $\mathrm{T} \rightarrow \mathrm{H} \rightarrow \mathrm{T}$ sites within these very top layers is not energetically feasible. Probably, interstitial motion in these cases takes place via different diffusion mechanisms which we are presently investigating.

\section{Conclusions}

Formation energies of interstitials and their diffusion characteristics were analyzed for silicon systems under uniaxial strain conditions. For both $\mathrm{T}$ and $\mathrm{H}$ sites, the interstitial formation process is energetically more favorable under tension but less favorable under compressive strain. The trend is similar for strains in $x-, y-$ and z-directions. Results indicate that strain applied in the direction perpendicular to a diffusing interstitial ( $y$-direction) has a minimal or no effect on $\Delta E$, the barrier height. In $x$ - and $z$-directions, on the other hand, tension increases $\Delta E$, and compression was found to reduce $\Delta E$ and thus to facilite the diffusion process. Furthermore, present calculations.show that the relative formation energy of an interstitial decreases within the surface region. This is partially because of the configurational changes (such as dimerization and the multilayer relaxation) which take place within the exposed surface region. At the same time, however, the barrier height for the interstital diffusion process via tetragonal $\rightarrow$ hexagonal $\rightarrow$ tetragonal sites increases as the defect nears the exposed surface region. 


\section{Acknowledgments}

This work was supported by NASA Ames Research Center through a prime contract NAS2-14031 to Eloret. 


\section{References}

[1] M. J. Aziz, J. Appl. Phys. Lett., 70, 2810 (1997).

[2] S. Coffa, A. La Magna, V. Privitera and G. Mannino, J. Appl. Phys. Lett., 73, 1571 (1998).

[3] C. S. Ozkan, W. D. Nix and H. Gao, J. Appl. Phys. Lett., 70, 2247 (1997); Mat. Res. Soc. Symp. Proc.,440, 323 (1997); and 442, 373 (1997).

[4] M. Tang, L.Colombo, J. Zhu and T. Diaz de la Rubia, Phys. Rev. B, 55, 14279 (1997).

[5] H. Balamane, T. Halicioglu and W. A. Tiller, Phys. Rev. B, 46, 2250 (1992).

[6] A. P. Smith, J. K. Wiggs, H. Jonsson, H. Yan, L. R. Corrales, P. Nachtigail and K. D. Jordan, J. Chem. Phys., 102, 1044 (1995).

[7] J. Zhu, Mat. Res. Soc. Symp. Proc. Vol. 469, 151 (1997).

[8] A. Antonelli and J. Bernholc, Phys. Rev. B, 40, 10643 (1989).

[9] P. Kringhoj, A. N. Larsen and S. Y. Shirayev, Phys. Rev. Lett., 76, 3372 (1996).

[10] Y. Zhao, M. J. Aziz, S. Mitha and D. Schiferl, Mat. Res. Soc. Symp. Proc. Vol. 442, 305 (1997). 


\section{Figure Captions}

Figure 1. Three-dimensional views of (a) tetragonal and (b) hexagonal sites. The darker sphere represents the interstitial $\mathrm{Si}$ atom, and bonded light color spheres are the lattice Si atoms.

Figure 2. Schematic side view representation of the Si crystal depicting the $x z$ plane. The dotted line shows the diffusion path. Open and solid circles represent tetrahedral and hexagonal sites, respectively.

Figure 3. Per cent variation in energy, $P_{E}$, for a tetragonal-site interstitial versus the applied strain. Solid, dashed and dotted lines show variations in $P_{E}$ for strain applied in the $x$-, $y$ - and $z$-directions, respectively. Negative and positive values of strain represent compression and tension, respectively.

Figure 4. Per cent variation in energy, $P_{E}$, for a hexagonal-site interstitial versus the applied strain. Solid, dashed and dotted lines show variations in $P_{E}$ for strain applied in the $x$-, $y$ - and $z$-directions, respectively. Negative and positive values of strain represent compression and tension, respectively.

Figure 5. Per cent variation in the barrier height, $P_{E}(B)$, for an interstitial diffusion as a function of applied strain. Solid, dashed and dotted lines show variations in $P_{E}(B)$ for strain applied in the $x$-, $y$ - and $z$-directions, respectively. Negative and positive values of strain represent compression and tension, respectively.

Figure 6. Variations in the interstitial formation energy as a function of the distance, $d_{z}$, from the exposed surface. Relative energy values with respect to bulk are plotted on the ordinate versus $d_{z}$. The values of $d_{z}$ are given in $\AA$.

Figure 7. Variations in the diffusion barrier height as a function of the distance, $d_{x}$, from the exposed surface. Relative values of the barrier heights with respect to the bulk value are plotted versus $d_{z}$. The values of $d_{z}$ are given in $\AA$. 
Figure 1

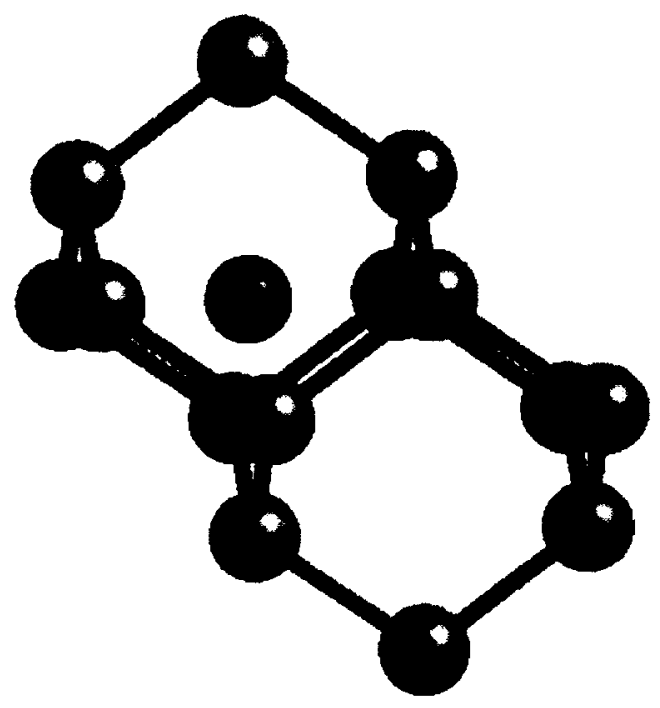

(a)

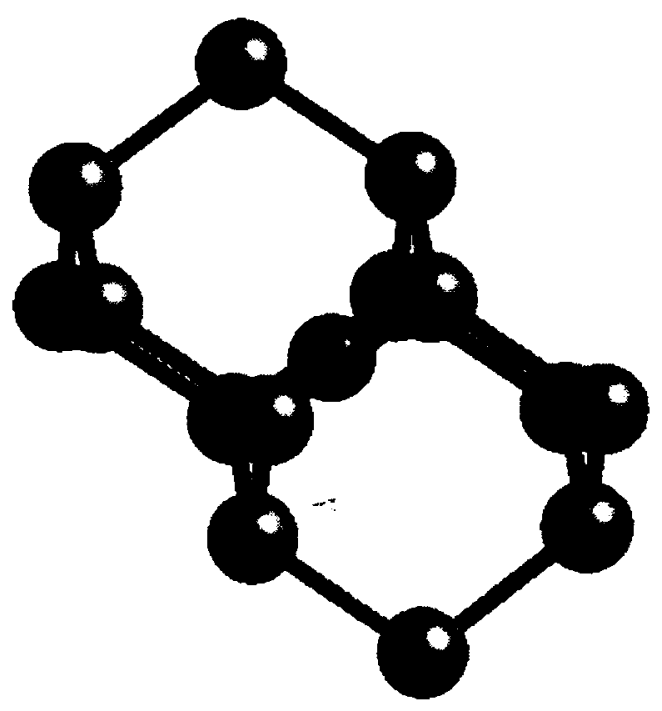

(b) 
Figure 2.

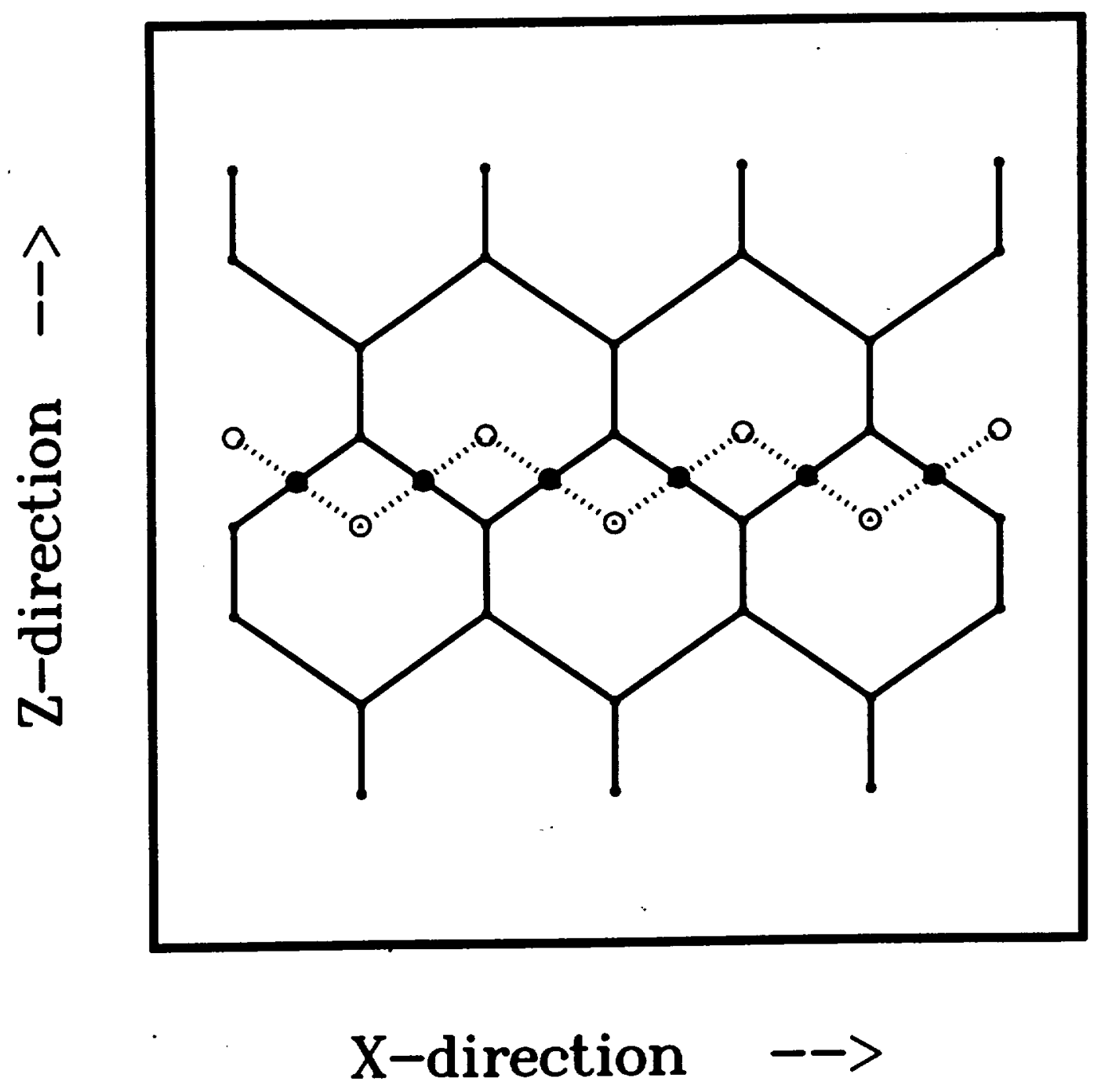


Figure 3.

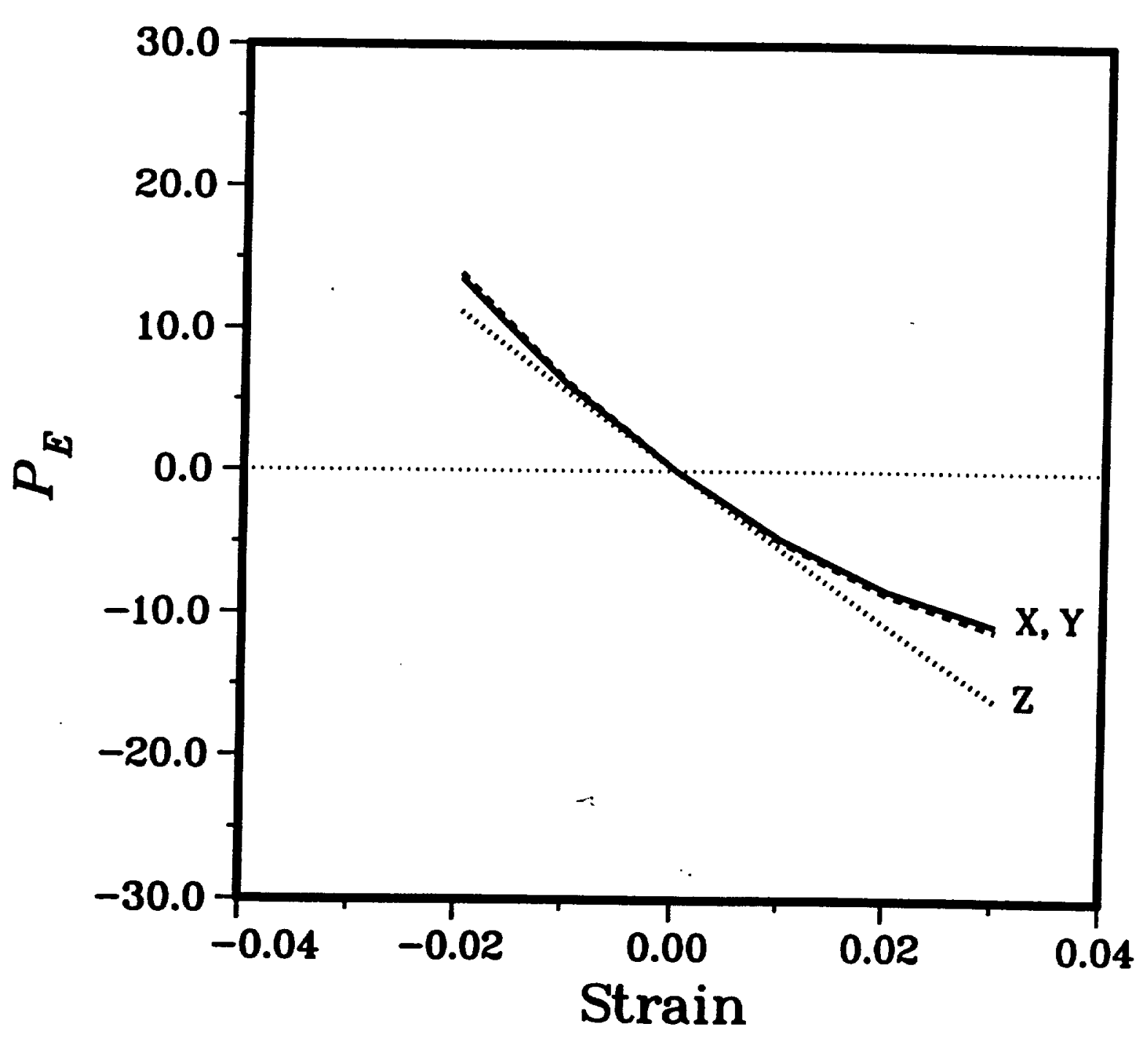


Figure 4.

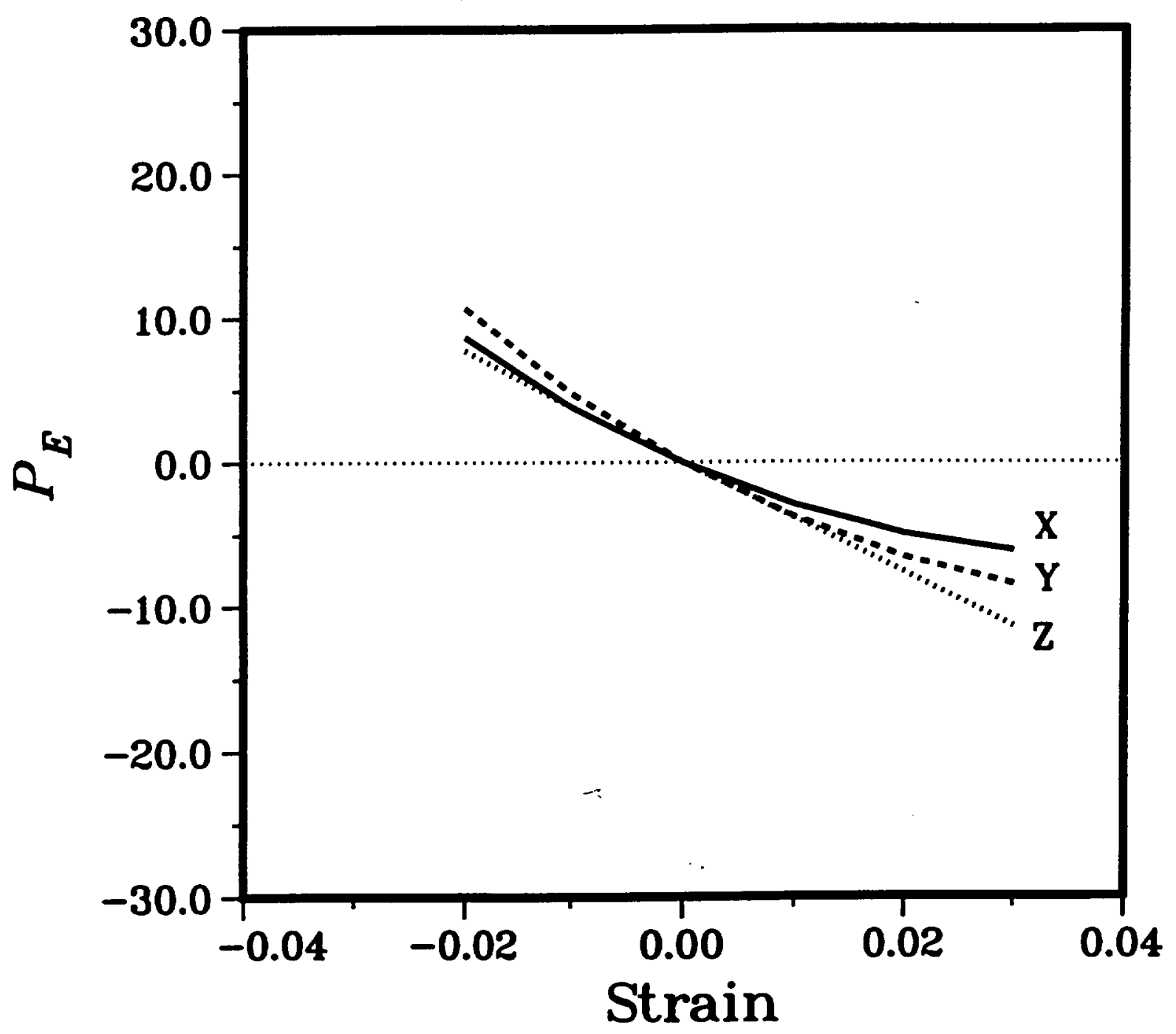


Figure 5.

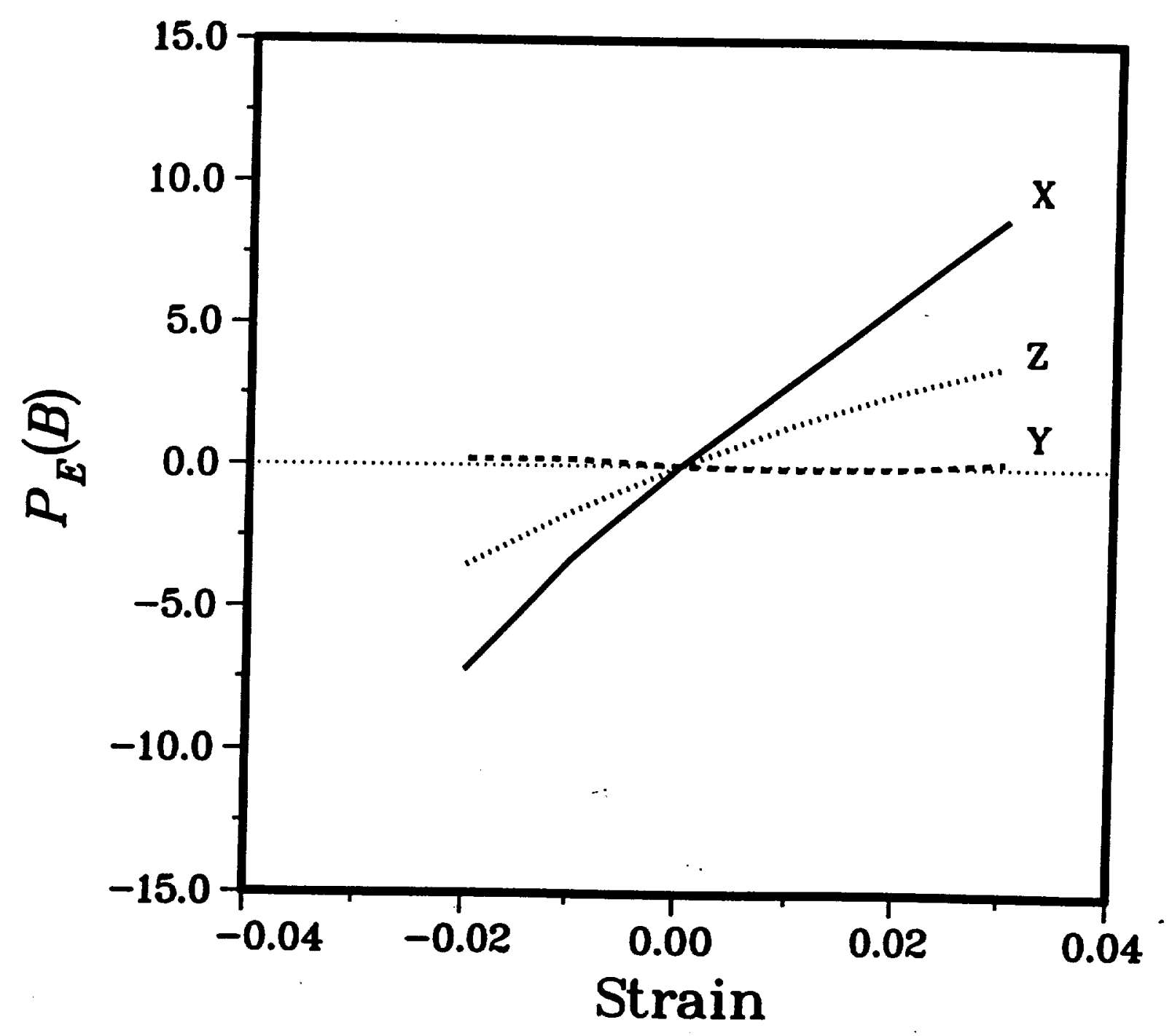


Figure 6.

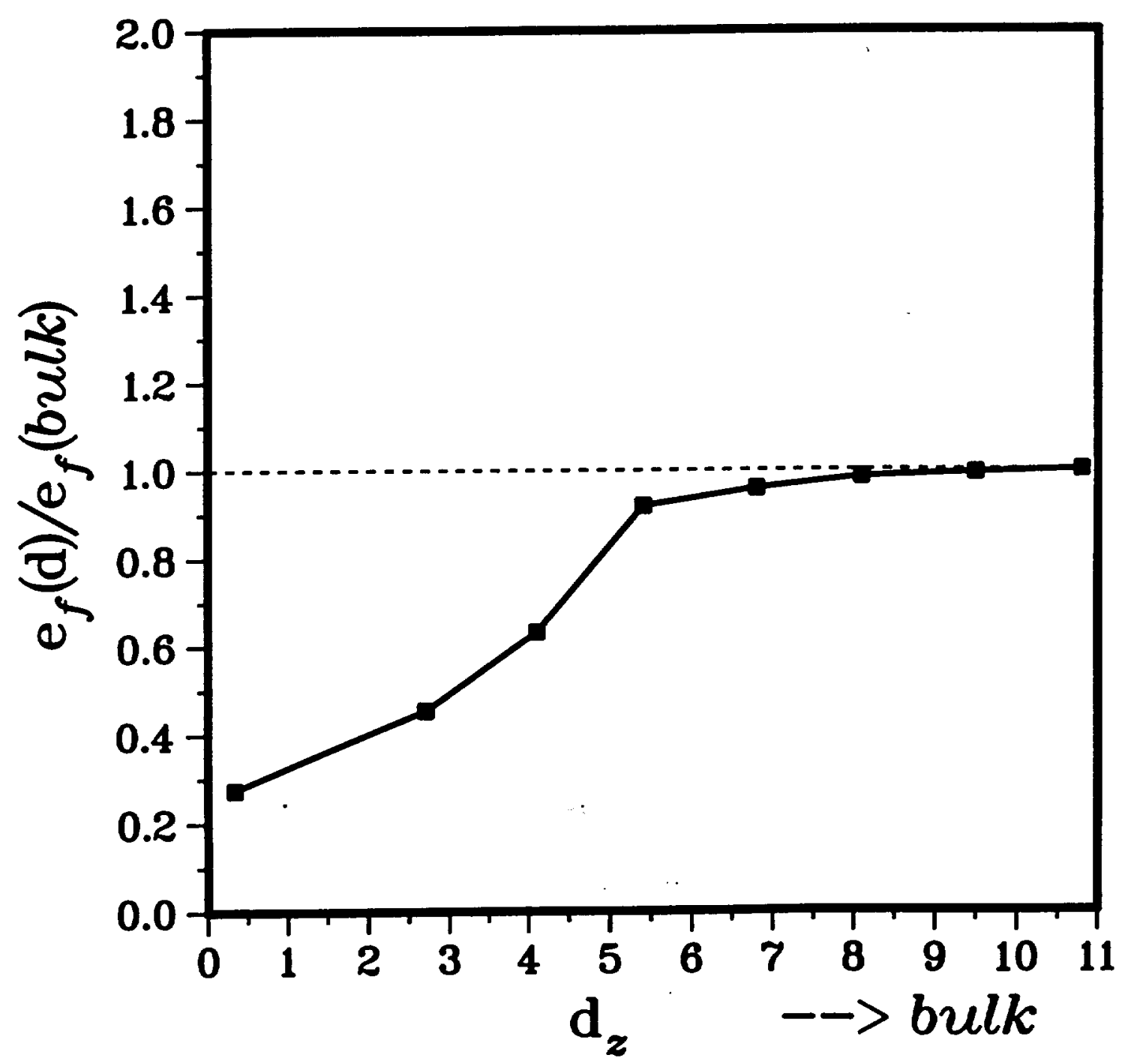


Figure 7.

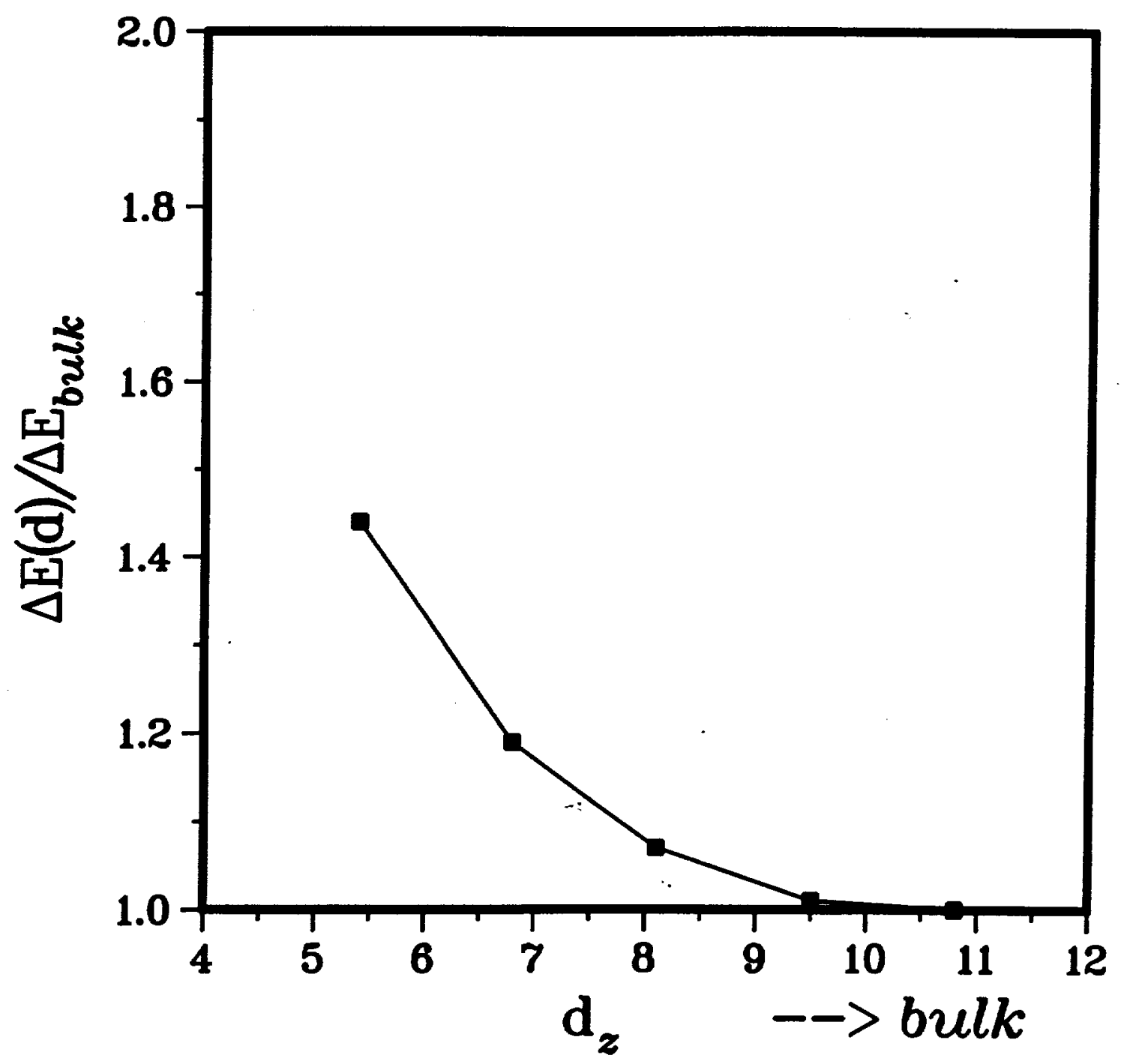

